\title{
O papel do preceptor no ensino médico e o modelo Preceptoria em um Minuto
}

\section{The preceptor's role in medical teaching and the One-Minute Preceptor Model}

\author{
Diego Chemello ${ }^{\mathrm{I}}$ \\ Waldomiro Carlos Manfrói ${ }^{\mathrm{I}}$ \\ Carmen Lúcia Bezerra Machado ${ }^{\mathrm{I}}$
}

\section{PALAVRAS-CHAVE}

- Educação de Graduação em Medicina

- Educação Médica

- Tutoria

- Hospitais de ensino

\begin{abstract}
R E S U M O
O ensino médico é extremamente importante na determinação da qualidade técnica dos futuros profissionais. Embora o Brasil apresente legislação específica sobre o tema, observamos discrepâncias no processo de aprendizado, o que, muitas vezes, compromete a formação dos futuros médicos. Além disso, métodos de ensino tradicionais, baseados fundamentalmente no diagnóstico de problemas, são ainda praticados em várias faculdades. Estes métodos relegam preceitos educacionais e, muitas vezes, colocam os estudantes em papel secundário. Nessa perspectiva, apresentamos um promissor método de ensino médico chamado Preceptoria em um Minuto (One-Minute Preceptor), salientando seu papel voltado ao profissional em formação. Destacamos também a importância deste método por permitir o ensino em tempo relativamente limitado, frente à crescente demanda por atendimentos.
\end{abstract}

\section{A B S T R A C T}

Medical teaching is extremely important for determining the quality of future professionals. Although Brazil has specific legislation on the subject, discrepancies have been observed in the learning process, often jeopardizing the training of future physicians. Furthermore, traditional teaching methods, based mainly on the diagnosis of problems, are still practiced in various medical schools. These methods ignore fundamental rules of education and relegate students to a secondary role. From this perspective, we present a promising method for medical education called the One-Minute Preceptor, emphasizing its role for in-training medical professionals. We also emphasize its importance for teaching in a time-limited scenario, due to the growing demand for care. 


\section{INTRODUÇÃO}

O ensino da prática médica a estudantes e residentes é um processo imprescindível à formação de futuros profissionais. Entretanto, estudos sobre o ensino da prática médica ambulatorial têm mostrado inadequações na qualidade e no tempo destinado pelos preceptores aos estudantes ${ }^{2}$. Há muitos anos o mercado de trabalho vem exigindo a formação de profissionais médicos hábeis em realizar diagnósticos e tratamentos em tempo cada vez mais limitado.

Com isso, professores de medicina em todo o mundo têm se empenhado na busca de estratégias de ensino que possam se adequar às novas demandas do século 21 . Não obstante, o enorme contingente de faculdades de Medicina existentes vem dificultando o desenvolvimento destas novas estratégias de preceptoria, na medida em que as turmas são constituídas por um número crescente de alunos, todos sob a tutela de um único preceptor.

Deste modo, a busca de preceptores capacitados ao ensino efetivo deste grande contingente de alunos é um problema iminente e preocupante. Em teoria, todas as escolas de Medicina do Brasil devem dispor de profissionais qualificados e capacitados para exercerem a tutela de alunos de graduação e médicos residentes. É o que estabelece inclusive a Resolução do Conselho Nacional de Residência Médica no 005/2004, de 8 de junho de 2004:

\footnotetext{
“Art. 2․ O preceptor/tutor terá a atribuição de orientar diretamente os médicos residentes do programa de treinamento. $\S 1^{\mathrm{o}}$ Haverá um preceptor/tutor para cada programa de Residência Médica. $\S 2^{\circ}$ A carga horária do preceptor/tutor será de 40 (quarenta) horas semanais, em tempo integral, com folga de um dia, preferencialmente aos domingos".
}

Entretanto, é sabido que a realidade está muito aquém dos preceitos definidos pela lei específica. Não dispomos de dados concretos no Brasil sobre número de preceptores e tempo destinado efetivamente por eles ao ensino de acadêmicos e médicos residentes. No entanto, podemos observar a série de movimentos reivindicatórios dos médicos residentes ocorridos nos últimos anos, em que a ausência de preceptores é destacada como fator importante na redução da qualidade do ensino médico brasileiro. Em entrevista recentemente realizada ao Conselho Regional de Medicina do Estado de São Paulo (Cremesp), Antônio Carlos Lopes - professor titular da Unifesp, diretor do Departamento de Residência Médica da Secretaria Nacional de Ensino Superior (Sesu) do Ministério da Educação e secretário-executivo da Comissão Nacional de Residência Médica (CNRM) - res- pondeu, ao ser questionado sobre a ausência do preceptor como fator prejudicial no ensino da residência médica:

É fundamental que se entenda que o ensino médico não
é apenas um repasse de conhecimentos. O professor, o
preceptor precisa estar ao lado do aluno, porque é a ati-
tude do profissional experiente, sua relação com o paci-
ente, que forma um médico mais humano e capaz de um
diagnóstico correto. De nada adianta um recurso tecno-
lógico avançado sem a presença de um médico que ouça
o paciente e saiba identificar a doença. Ao contrário, um
médico bem formado resolve qualquer crise sem a tec-
nologia. O residente deve saber tomar decisões, mas
sempre sob a supervisão do preceptor, que precisa estar
presente. Só se aprende medicina com o testemunho da
presença.

Estas informações, que podem parecer a simples opinião de um profissional, podem ser comprovadas por alguns estudos que demonstram a importância do preceptor em medicina não somente para os alunos, mas também para a melhora da qualidade assistencial prestada aos pacientes. Recentemente foi observado que, quando o médico é acompanhado por uma equipe de acadêmicos ao exercer a função de preceptor, o paciente recebe adicionais 12,4 minutos de atenção pela equipe médica, embora o tempo do preceptor com o paciente seja reduzido, em média, em 30 segundos.

Portanto, entendemos que a formação de preceptores com habilidade e competência para transmitir (de forma eficaz) conhecimentos em curto espaço de tempo é tarefa cada vez mais desafiadora. No Modelo de Ensino Tradicional (MET) ainda amplamente utilizado, toda atenção é centrada na figura do paciente e do preceptor. Neste modelo, o processo de aprendizado ambulatorial apresenta três componentes essenciais: (1) a apresentação do caso pelo aluno; (2) perguntas feitas pelo preceptor com o objetivo de buscar informações adicionais; (3) discussão do caso em grupo e definição da conduta. Isto pode consumir até três quartos de todo o tempo de ensino com a consulta médica per se, prejudicando o processo de ensino por redução do tempo destinado à discussão do caso e resolução de dúvidas. Além disso, a retroalimentação (feedback) dos alunos, parte essencial para o processo de aprendizado, fica sobremaneira prejudicada.

Em nossa opinião, essa abordagem clássica de ensino médico ignora as mudanças ocorridas no perfil dos estudantes nos últimos anos e pode ser um fator importante para a redução do interesse do aluno e a consequente queda de seu aprendizado. Ademais, as perguntas usualmente feitas pelos orientadores no MET são direcionadas basicamente ao correto diagnóstico do caso, relegando a segundo plano o processo de aprendizado e os 
interesses do aluno. Nesse sentido, questões como: "Qual é a saturação de oxigênio do paciente?" ou "É a dor mais forte já sentida por este paciente?" limitam o poder do aluno de deliberar sobre o caso, ao mesmo tempo em que abandonam quase por completo a preocupação com o ensino.

De acordo com os fatores citados, nos parece que o MET não é mais inquestionável e absoluto como se pensava em anos anteriores. É então plenamente justificável a implementação de novas estratégias de ensino, mais dinâmicas e participativas, que busquem a otimização do tempo e o aumento da eficácia no processo de ensino. Acreditamos ser de fundamental valor o incremento da interação professor-aluno e a troca de conhecimentos entre ambos, desenvolvendo uma relação bilateral e voltada para o ensino, sem esquecer o cuidado com o paciente. Nessa perspectiva, o ensino interativo baseado em casos aumenta a incorporação de informações necessárias aos estudantes. O feedback dos preceptores, igualmente, mostra-se fundamental na melhora do aprendizado dos estudantes e residentes.

Buscando novas propostas de ensino com as características acima citadas, Neher e cols., do Departamento de Medicina de Família da Universidade de Washington, apresentaram pela primeira vez um novo modelo de preceptoria em ambulatórios ${ }^{14,15}$. A base teórica deste modelo possibilita ao preceptor transmitir a seus alunos, em curto espaço de tempo e de maneira eficaz, valiosas informações médicas, ao mesmo tempo em que lhe permite melhor conhecimento das características do grupo de estudantes. Este modelo de ensino médico recebeu a denominação de Preceptoria em um Minuto (One-Minute Preceptor) (OMP) e foi desenvolvido para ser usado quando um estudante ou residente, após avaliar um caso clínico, solicita auxílio de seu preceptor para a solução de um ou mais aspectos. O método envolve a elaboração de cinco etapas fundamentais em forma de questionamentos (denominados microskills), que apresentamos a seguir: comprometimento com o caso; busca de evidências concretas; ensine regras gerais; reforce o que está correto; corrija os potenciais erros.

\section{Comprometimento com o caso}

Após um estudante apresentar um caso, ele geralmente espera de seu preceptor algum comentário a respeito do que poderia estar acontecendo. Entender como este estudante interpreta o caso em questão é o primeiro passo na OMP. Questionamentos amplos (perguntas abertas) permitem ao estudante mostrar sua interpretação do caso, ao mesmo tempo em que o incentiva a discutir sobre os principais aspectos do mesmo. Perguntas do tipo: "O que você acha que está acontecendo com o paciente?" ou "Qual a sua ideia sobre a conduta a ser tomada?" são exemplos a seguir. Ao contrário, perguntas específicas ou comentários desestimulantes devem ser evitados, pois inibem a manifestação espontânea do aluno e limitam o espaço para futuras discussões.

\section{Busca de evidências concretas}

O passo seguinte a ser observado pelo preceptor, antes mesmo de expressar sua opinião, é questionar ao aluno os motivos que o levam a definir tal diagnóstico ou conduta. Assim, permitimos que o estudante expresse seus conhecimentos vigentes sobre determinada doença, bem como seu grau de entendimento sobre ela. Ao mesmo tempo, o preceptor identifica o enfoque que poderá dar ao caso, segundo a perspectiva de ensino. As perguntas mais frequentes a empregar nesta etapa são também de caráter amplo e representadas pelos seguintes exemplos: "Por que você acha isso?" ou "O que mais você considera a respeito deste caso?".

\section{Transmita regras de ensinamento gerais}

Já está comprovado que o conhecimento sobre determinado assunto é mais bem transmitido e memorizado quando são introduzidas regrais gerais e preceitos amplos, ao invés de informações detalhadas. O melhor caminho ao bom preceptor é iniciar com conhecimentos básicos e progredir com assuntos mais complexos, à medida que as habilidades dos alunos também aumentam. Com isso, o desafio para a pesquisa e o acréscimo de conhecimento também se desenvolvem, e o ensino pode continuar até mesmo sem o contato direto com o preceptor.

\section{Enfatize o que está correto}

Mostrar ao estudante o que está correto, provendo-lhe feedback positivo, é uma excelente maneira de aumentar sua autoconfiança e estimulá-lo a buscar novos conhecimentos. O preceptor deve enfatizar o que foi feito de correto na apresentação do caso ou na conduta com frases do tipo: "Vejo que sua capacidade de síntese melhorou em relação aos casos antes apresentados" ou "A escolha do tratamento apresentada me parece bastante adequada ao caso em questão" são exemplos de frases bem empregadas nesse modelo.

\section{Corrija o que está incorreto}

Tão importante quanto as etapas anteriores, a correção dos erros é um processo fundamental para o processo de aprendizado. Isto deve ser realizado assim que possível dentro do processo de discussão do caso. Já está comprovado que erros não corrigidos apropriadamente têm maior tendência a se repetirem. Obviamente, todo esforço deve ser feito para tornar esta correção o menos desagradável possível, enfatizando a discussão aberta e pra- 
zerosa. Sugestões para a busca de novas estratégias, abertura para a possibilidade de uma autocrítica, bem como sugestões de leituras específicas para discussões posteriores são excelentes maneiras de correção de erros neste processo.

Um dos erros mais comuns que observamos junto aos preceptores, especialmente os mais jovens e inexperientes, é que eles buscam transmitir o maior número possível de informações em curto espaço de tempo. O modelo de OMP enfatiza exatamente o contrário, ou seja, a transmissão limitada de informações e sempre de acordo com os casos apresentados. Isto facilita o aprendizado dos alunos e os estimula a buscar novas fontes de pesquisas sobre determinado assunto.

\section{MEDICINA BASEADA EM EVIDÊNCIA E O MODELO OMP}

No final da década de 1980, os professores do Departamento de Epidemiologia Clínica e Bioestatística da Universidade McMaster David Sackett e Gordon Guyatt iniciaram o movimento conhecido hoje como Medicina Baseada em Evidência (MBE). Desde então, a medicina vivenciou uma das maiores mudanças de paradigmas até hoje ocorridas: estudos com metodologias adequadas e ao mesmo tempo eficazes tornaram-se imprescindíveis à validação de novos métodos de diagnóstico e tratamento. Felizmente, a área da educação médica vem incorporando progressivamente os conceitos da MBE com o objetivo de aprimorar as metodologias de ensino na área da saúde. Entretanto, grande parte das informações relacionadas a métodos de preceptoria, satisfação dos alunos e desempenho dos professores é ainda limitada por estudos com amostra relativamente pequena e metodologia, muitas vezes, inadequada.

Nesta seção analisaremos a informação disponível sobre eficácia e efetividade do método OMP, quando comparado a outros métodos de preceptoria (tradicionalmente mais usados) aplicados na prática médica.

No estudo originalmente publicado por Neher e cols., foi relatado que o OMP, quando ensinado a médicos residentes, tem a capacidade de se incorporar à prática destes profissionais por tempo relativamente adequado: 26 dos 29 médicos residentes treinados em OMP continuaram usando $90 \%$ de seus preceitos em encontros posteriores com alunos, por um período médio de quatro anos. Huang e cols. mostraram que até mesmo preceptores que nunca haviam recebido treinamento formal no método usaram alguns de seus preceitos em atividades de preceptoria. Segundo dados analisados pelos autores em 86 encontros com 12 preceptores, as três primeiras perguntas (microskills) foram usadas em $40 \%$ a 60\%. Entretanto, o feedback positivo e a correção dos erros foram menos usados (12,8\% e 18,6\%, respectivamente). Salientamos nesses estudos o pequeno tamanho amostral e a ausência de grupo controle, o que limita as conclusões sobre o método de ensino.

Em estudo realizado por Aagaard e cols., observou-se que o OMP mostrou eficácia e efetividade consideradas pelo menos semelhantes às do MET. Neste último, observou-se maior foco de atenção no diagnóstico correto do caso e menos participação dos estudantes no processo. Por outro lado, os preceptores que analisaram casos pelo OMP deram mais importância a diagnósticos diferenciais, a métodos diagnósticos e à apresentação natural da doença, o que, em nosso ponto de vista, torna o processo de ensino mais eficaz e fundamentalmente voltado ao estudante.

Em estudo prospectivo que submeteu preceptores e residentes de terceiro ano a workshops em OMP, foi observado um aumento do aprendizado por parte dos alunos $(p=0,03)$. Houve também a impressão, por parte dos preceptores, de que os alunos aumentaram a capacidade de chegarem às suas próprias conclusões sobre o caso $(\mathrm{p}=0,001)$ e estabeleceram planos para as próximas preceptorias $(\mathrm{p}=0,02)$. Esses últimos achados são extremamente interessantes, na medida em que o OMP pode ser um método de ensino que estimula mais o processo de autoaprendizado e o estudo fora do ambiente profissional.

Em ensaio clínico randomizado (ECR), residentes de Medicina Interna da Universidade de Michigan foram randomizados para dois grupos: intervenção, com aulas com treinamento OMP ( $n=28)$ e grupo controle (MET) ( $n=29)$. Os residentes alocados para o grupo intervenção recebiam aulas e treinamento prático em sessões de uma hora, durante o período de almoço. Após o período destinado a treinamento, os residentes que foram submetidos ao OMP mostraram maior motivação, feedback e comprometimento para o ensino de alunos de graduação, embora parâmetros de desempenho destes em processo de ensino não tenham sido testados. Apesar do pequeno tamanho amostral, esse estudo representa a primeira tentativa de testar o OMP por meio de ECR, considerado uma das melhores evidências disponíveis na literatura médica.

\section{CONCLUSÕES}

A despeito das limitações, concluímos que o OMP é prático, facilmente aplicável no dia a dia e vem encontrando boa aceita- 
bilidade pelos profissionais que com ele adquirem algum contato.

Evidências recentes da literatura têm mostrado que o modelo tem eficácia e efetividade pelo menos semelhantes às do MET, com algumas vantagens relacionadas ao estímulo dos alunos para o aprendizado.

Em nossa opinião, novos estudos prospectivos com adequada metodologia e poder estatístico são necessários para definir a aplicabilidade mais apropriada do OMP, inclusive em nosso país. Apesar destes fatos, acreditamos ser este um método de grande utilidade, especialmente com as mudanças que o ensino da medicina vem apresentando.

\section{REFERÊNCIAS}

1. Aagaard E, Teherani A, Irby DM. Effectiveness of the One-Minute Preceptor Model for diagnosing the patient and the learner: Proof of Concept. Acad Med. 2004;79(1):42-29.

2. Irby D. Teaching and learning in ambulatory care settings: a thematic review of the literature. Acad Med. 1995;70:898-931.

3. Brasil. Ministério da educação. Secretaria de Educação Superior. Comissão Nacional de Residência Médica. Resolução $\mathrm{n}^{\circ}$ 5, de 8 de junho de 2004. Dispõe sobre os serviços de preceptor / tutor dos programas de Residência Médica. Diário Oficial da União. Brasília, № 111, 11 jun. 2004; Seção 1, p. 18-19.

4. Lopes AC. Sem preceptor não há residência médica [entrevista]. Jornal do Cremesp 2007, Abr. 236; p. 3-4

5. Usatine RP, Tremoulet PT, Irby D. Time-efficient preceptors in ambulatory care settings. Acad Med. 2000;75:639-42.

6. Knudson MP, Lawler FH, Sqeig SC, Moreno CA, Hosokawa MC, Black RL. Analysis of resident and attending physician interactions in family medicine. J Fam Pract. 1989;28: 705-9.

7. Irby DM, Ramsey P, Gillmore J, Schaad D. Characteristics of effective clinical teachers of ambulatory care medicine. Acad Med. 1991;66:54-5.

8. Loftus TH, McLeod PJ, Snell LS. Faculty perceptions of effective ambulatory care teaching. J Gen Intern Med. 1993;8:575-77.

9. O'Malley PG, Kroenke K, Ritter J, Dy N, Pangaro L. What learners and teachers value most in ambulatory educational encounters: a prospective, qualitative study. Acad Med. 1999;74:186-91.

10. Irby D, Bowen JL. Time-efficient strategies for learning and performance. The Clinical Teacher. 2004;1(1):23-8.
11. Elstein AS, Shulman LS, Sprafka SA, al e. Medical problem solving: an analysis of clinical Reasoning. Cambridge (MA): Harvard University Press; 1978.

12. Raskind HS. Brousseau effective feedback and evaluation in clinical medicine. Special: Office-based medical student teaching. The One-Minute Preceptor. 2001;5(2):36-8.

13. Ferenchick G, Simpson D, Blackman J, DaRosa D, Dunnington $G$. Strategies for efficient and effective teaching in the ambulatory care setting. Acad Med. 1997;72:277-80.

14. Neher JO, Stevens NG. The One-Minute Preceptor: shaping the teaching conversation. Fam Med 2003;35:391-3.

15. Neher JO, Gordon KC, Meyer B, Stevens N. A five-step "microskills" model of clinical teaching. J Am Board Fam Pract. 1992;5:419-24.

16. Parrott S, Dobbie A, Chumley H, Tysinger JW. Evidence-based office teaching - the five-step microskills model of clinical teaching. Fam Med. 2006;38:164-7.

17. Bowen JL, Irby D. Assessing quality and costs of education in the ambulatory setting: a review of the literature. Acad Med. 2002;77:621-80.

18. Huang WY, Dains JE, Monteiro FM, Rogers JC. Observations on the teaching and learning occurring in offices of community-based family and community medicine clerkship preceptors. Fam Med. 2004;36:131-6.

19. Irby DM, Aagaard E, Teherani A. Teaching points identified by preceptors observing One-Minute Preceptor and traditional preceptor encounters. Acad Med 2004;79:50-5.

20. Salerno SM, O'Malley PG, Pangaro L, Wheeler GA, Moores LK, Jackson JL. Faculty developed seminars based on the One-Minute Preceptor improves feedback in the ambulatory setting. J Gen Intern Med. 2002;17:779-87.

21. Furney SL, Orsini AN, Orsetti KE, Stern DT, Gruppen LD, Irby D. Teaching the One-Minute Preceptor - a randomized controlled trial. J Gen Intern Med. 2001;16:620-4.

\section{CONTRIBUIÇÃO DOS AUTORES}

A autora Carmem Lúcia B. Machado contribuiu na concepção inicial da idéia (juntamente com os demais autores), procurando centralizar o tema também no papel do educador no ensino da prática médica. Contribuiu também com a bibliografia e foi responsável pela revisão do mesmo.

Diego Chemello contribuiu na concepção da idéia, revisão da literatura e seleção crítica dos estudos publicados sobre o assunto e, após discussão com os demais autores, foi responsável pela redação final do manuscrito. 
Waldomiro Carlos Manfrói foi responsável pela análise critica da bibliografia pré-selecionada, determinado os artigos mais apropriados e em concordância com o tema proposto. Após elaboração do manuscrito, foi responsável pela revisão inicial do manuscrito.

\section{CONFLITO DE INTERESSES}

Declarou não haver.

\section{ENDEREÇO PARA CORRESPONDÊNCIA}

Waldomiro Carlos Manfrói

Rua Ramiro Barcelos, 2400 - 4o andar

Bairro Bom Fim - Porto Alegre

CEP 90035-903 RS

E-mail:wmanfroi@hcpa.ufrgs.br 